\title{
Analysis of base characteristics of trench gate field termination IGBT
}

\author{
Wang $\mathrm{Bo}^{1, *}$ \\ ${ }^{1}$ Wuhan Donghu University, CHINA
}

\begin{abstract}
Trench gate structure represents the latest structure of Insulated Gate Bipolar Transistor(IGBT). Because there are great differences in model analysis coordinate system and carrier transport between trench gate structure and planar gate structure, the modeling method using planar gate structure will inevitably have great deviation. Based on the characteristics of trench gate structure and model analysis coordinate system, the base region is divided into PNP and PIN by considering the two-dimensional effect of carriers. According to whether the trench of PIN part can be covered by depletion layer of PNP part, the specific base region current is analyzed. Finally, simulation and experimental verification are carried out.
\end{abstract}

\section{Introduction}

Insulated Gate Bipolar Transistor(IGBT) is a kind of composite device which combines the structure of field effect transistor and bipolar transistor, and absorbs their advantages at the same time. It is widely used in various medium and high power electronic devices. With the continuous improvement of the design level and manufacturing process of power semiconductor devices, the internal gate structure of IGBT has changed from the early planar gate to the later trench gate ${ }^{[1]}$. This trench gate structure absorbs the advantages of previous generations, and adopts some new structures and technologies. Its performance indexes, such as withstand voltage capability, conduction current capability, on-state voltage drop and switch damage, have been significantly improved, and it has excellent comprehensive performance ${ }^{[2-3]}$.

Since the advent of IGBT in 1980s, many literatures have carried out relevant research on IGBT mathematical model, and the early theory of planar gate structure has been relatively mature. The most representative Hefner model studies the steady-state and transient mathematical models of planar gate structure by using the theories of bipolar carrier transport in base region, quasi-static approximation and transient carrier linear distribution, and the existing IGBT mathematical models are basically developed on the basis of this model ${ }^{[4-5]}$. However, this model only makes a one-dimensional approximation to the distribution of excess carriers in the base region, and seldom studies the influence of the change of trench gate structure on the two-dimensional distribution. Other literatures have adopted the method of two-dimensional distribution of carriers, which promotes the research of trench gate structure ${ }^{[6]}$.

In this paper, based on the characteristics of trench gate structure and model analysis coordinate system, by dividing the base region into PNP and PIN and considering the two-dimensional effect of carriers in transient process, the transient specificity of the base region current is analyzed according to whether the trench of PIN can be covered by depletion layer of PNP. Finally, simulation and experimental verification are carried out.

\section{Theoretical analysis}

\subsection{Trench gate IGBT model coordinate system}

The planar schematic diagram of trench gate field termination IGBT structure is shown in Figure 1, which is usually regarded as a BJT structure driven by MOSFET. The vertical width $L_{x}$ of the trench reflects the depth of the trench into the base region, and the horizontal width $L_{y}$ of the trench reflects the proportion of the base region occupied by the trench. Among them, the $\mathrm{P}$ body region on the upper part of the base region is very small relative to the width of the base region, which is similar to the planar gate type. While the trench gate on the upper part of the base region goes deep into the base region, and the depth can't be ignored compared with the width of the base region. Therefore, different from planar gate structure, trench gate structure needs to consider the influence of trench.

\footnotetext{
* Corresponding author: author@e-mail.org
} 


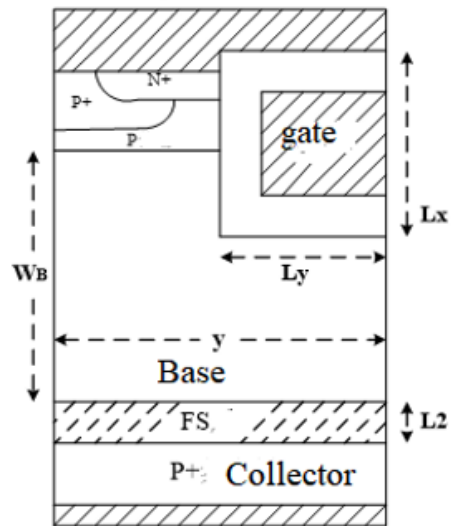

Fig1. Schematic diagram of trench gate IGBT structure

The mathematical model analysis coordinate system of trench gate field termination IGBT is shown in Figure 2. The horizontal axis of FS layer coordinate system is $x^{*}$, the horizontal axis of base area coordinate system is $x$, and the vertical axis of coordinate system is $y$. The width of base region is $W_{B}$, and the width of FS layer is $W_{H}$. According to the carrier movement characteristics of $\mathrm{p}$ body region and trench gate in the base region, the base region is divided into PNP at the lower part of $\mathrm{p}$ body region and PIN at the lower part of gate. The depletion layer width of PNP part is $W_{d e p, P N P}(t)$, and the quasineutral base region width is $W_{L}$. The electron current and hole current of PNP part and PIN part are calculated respectively, and then the electron current and hole current in the base region are summarized.

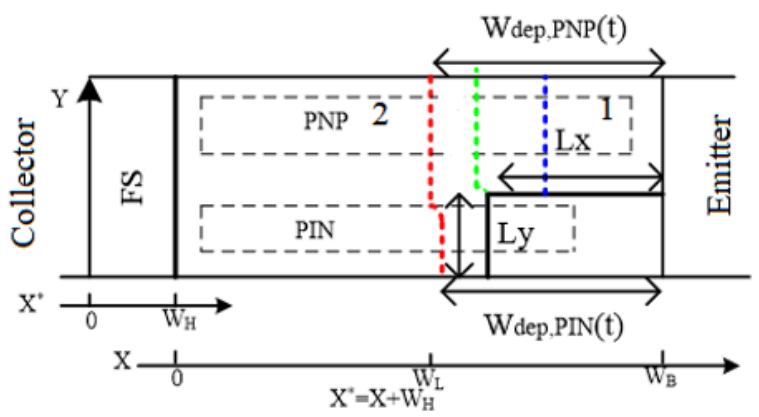

Fig2. Coordinate system of trench gate IGBT model

\subsection{Analysis of characteristics of base region}

Under IGBT switching transient, the excess carrier concentration in the base region meets the conditions of large injection and low gain, and the continuity equation under bipolar transport condition is:

$$
\frac{d^{2}(\delta p(x, t))}{d x^{2}}=\frac{\delta p(x, t)}{L_{L}^{2}}+\frac{1}{D_{L}} \frac{d(\delta p(x, t))}{d t}
$$

Where: $\delta p(x, t)$ refers to excess carrier concentration; $L_{L}$ is the base carrier bipolar diffusion length; $D_{L}$ is the bipolar diffusion coefficient of base carriers.

When the depletion layer in the PNP part of the base region widens to the PIN part, it bends due to the influence of the gate voltage. The width of the depletion layer formed by the collector-emitter voltage and the gate voltage in the PIN part is:

$$
W_{d e p, P I N}(t)=\sqrt{\frac{2 \varepsilon_{s i}\left(V_{b c}(t)-V_{G S}(t)+V_{T d}\right)}{q N_{L}}}
$$

In which: $\varepsilon_{s i}$ is the dielectric constant of silicon; $V_{b c}(t)$ is BJT base-emitter voltage drop; $V_{G S}(t)$ is the gate voltage; $V_{T d}$ is that threshold voltage of the gatedrain overlap depletion layer; $q$ is the electronic quantity; $\quad N_{L}$ is the base doping concentration.

According to whether the depletion layer of PNP part can be widened to cover the trench gate of PIN part, that is, the relationship between the width of depletion layer of PIN part and the width of trench gate deep into the base region, two cases are analyzed.

The first case: the depletion layer of PNP part failed to widen the trench gate area covering PIN part, that is $W_{\text {dep }, P I N}(t) \leq L_{x}$.

The depletion layer of PNP part expands along the Yaxis direction and fails to surround the trench area of PIN part, as shown in Figure 2, the depletion layer expands to the 1 position. Then the PNP part and PIN part are described separately, and then the electron current flowing into the trench and the hole current flowing into the cathode are synthesized. The movement process of carriers is understood as follows: electrons are injected from the trench gate and are continuously converted into holes in the process of moving to the FS layer; When holes are injected from the anode and move to the cathode after passing through the FS layer, the hole concentration in the PNP part decreases gradually, and is considered to be approximately zero at the depletion layer. The hole concentration in the PIN part decreases gradually similar to that in the PNP part, and turns to flow into the depletion layer along the trench wall at the trench.

For PNP, the depletion layer width and quasi-neutral width are:

$$
\left\{\begin{array}{l}
W_{d e p, P N P}(t)=\sqrt{\frac{2 \varepsilon_{s i} V_{c e}(t)}{q N_{L}}} \\
W_{L}(t)=W_{B}-W_{d e p, P N P}(t)
\end{array}\right.
$$

The two boundary conditions of excess carrier concentration are:

$$
\left\{\begin{array}{l}
\delta p(x=0, t)=P_{0}(t) \\
\delta p\left(x=W_{L}(t), t\right)=0
\end{array}\right.
$$

The carrier concentration distribution in the transient process is approximated by a moving linear distribution, and the expression of space-time distribution of excess carrier concentration in the base region is obtained. The distribution process can be represented as Figure 3 . 


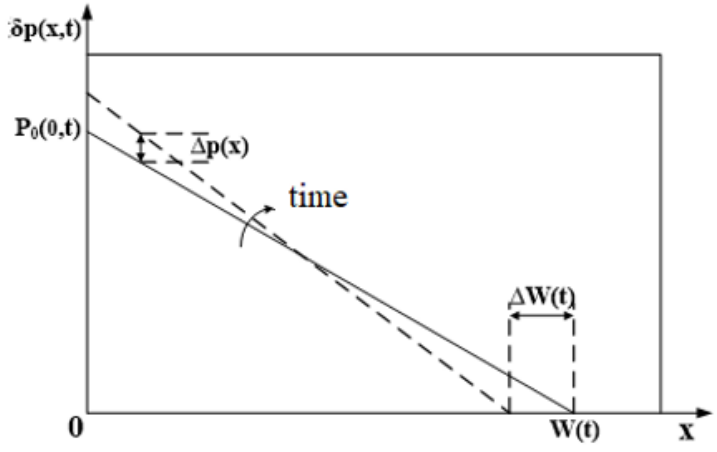

Fig3. Distribution of excess carrier concentration in base region during switching transient process

For the PIN part, the excess carrier concentration has the same distribution as that of PNP part in most areas of $\mathrm{X}$ axis. When near the trench, the excess carrier bends to PNP part and is swept into the depletion layer together with the excess carrier of PNP part. Therefore, the excess carrier concentration distribution of the PIN part is approximately considered to be the same as that of the PNP part at the same distance $\left(0 \sim\left(W_{B}-L_{x}\right)\right)$.

The quasi-neutral width of the PIN portion is determined by the width of the base region and trench metallurgy junction, and the excess carrier concentration distribution of the PIN portion has the same form as that of the PNP portion. The expressions of electron current and hole current in PNP part and PIN part are obtained by using bipolar transport equations of electron current and hole current respectively.

Among them, the currents of PNP part and PIN part satisfy:

$$
\frac{I_{P N P}}{I_{P I N}}=\frac{y-L_{y}}{L_{y}}
$$

The second case: the depletion layer of PNP part can widen the trench gate area covering PIN part, that is $W_{\text {dep,PIN }}(t)>L_{x}$.

The depletion layer formed by PNP expands in the Yaxis direction to cover the trench area of PIN. As shown in Figure 2, when the depletion layer expands to the 2 region, the expressions of carrier concentration distribution, electron current and hole current in the base region are obtained by a processing method similar to that of planar gate. The difference from planar gate is the calculation of junction capacitance of depletion layer under the gate.

The transient description of trench gate structure is similar to that of planar gate structure. The bipolar transport equation of electron current and hole current in base region is:

$$
\left\{\begin{array}{l}
I_{n L}(x, t)=\frac{b_{L}}{1+b_{L}} I+q D_{L} \frac{d(\delta n(x, t))}{d x} \\
I_{p L}(x, t)=\frac{1}{1+b_{L}} I-q D_{L} \frac{d(\delta p(x, t))}{d x}
\end{array}\right.
$$

The expression of hole current is obtained, and the hole current flowing into and out of the base region is obtained.

Therefore, for the above two cases, the hole current flowing into the base region and the hole current flowing out of the base region are obtained respectively.

\subsection{Simultaneous analysis of base area and FS layer}

The hole current and electron current flowing through the boundary between FS layer and base region are equal, that is, the current at the boundary is continuous, but the excess carrier concentration is discontinuous, as shown in Figure 4.

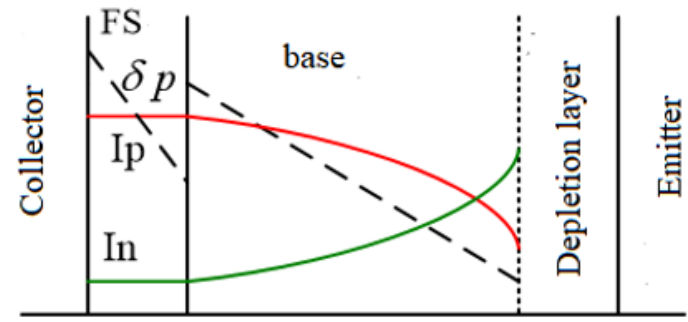

Fig4. Simultaneous analysis of base zone and FS layer

The carrier concentration at the boundary satisfies the relation:

$$
I_{p L}(0, t)=I_{p H}\left(x^{*}, t\right)
$$

According to whether the depletion layer of the base region can be widened to cover the trench gate region and the relationship between the total charge, the expression of the charge of the base region from the total charge is obtained.

Then, according to the quasi-thermal balance relationship between FS layer and base region and the current at the boundary, the relationship between the hole current and the total charge of internal BJT collector and base is obtained continuously, thus eliminating the intermediate quantity of the assumed boundary concentration.

\section{Experimental verification}

A trench gate field termination IGBT module with KW60N65S model is selected, and the rated voltage and current levels are $650 \mathrm{~V}$ and $60 \mathrm{~A}$ respectively. The experimental test conditions are as follows: the bus voltage is set to $400 \mathrm{~V}$, the driving voltage is $15 \mathrm{~V}$ when switching on, the load inductance is $500 \mathrm{uH}$, and the room temperature. Double pulse test method is used to test IGBT collector-generator voltage and collector current, and the IGBT turn-off waveform is shown in the Figure 5.

With the trench gate model established in this paper, the parameters required for simulation are provided by the device manufacturer, and the stray inductance of the line is set to $1 \mathrm{uH}$ according to the experimental test circuit. After the initial value is input, it is solved, and the turn-off transient simulation waveforms of IGBT are obtained as shown in the Figure 5. 


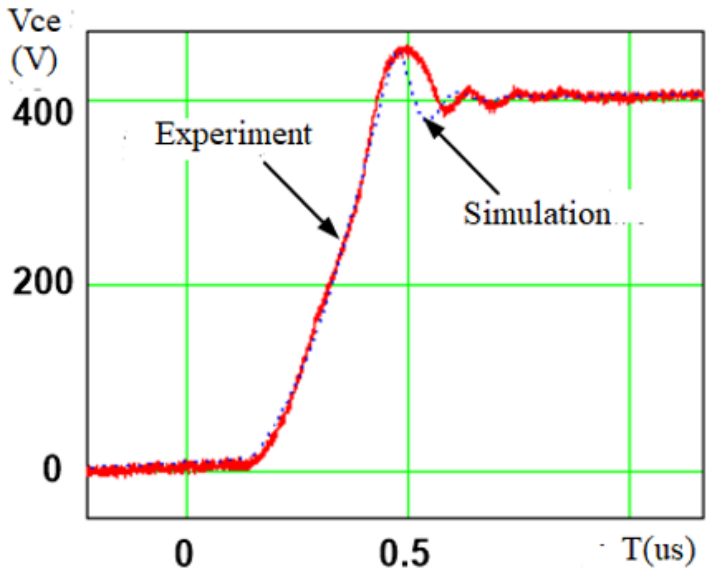

(a) Turn-off voltage waveform

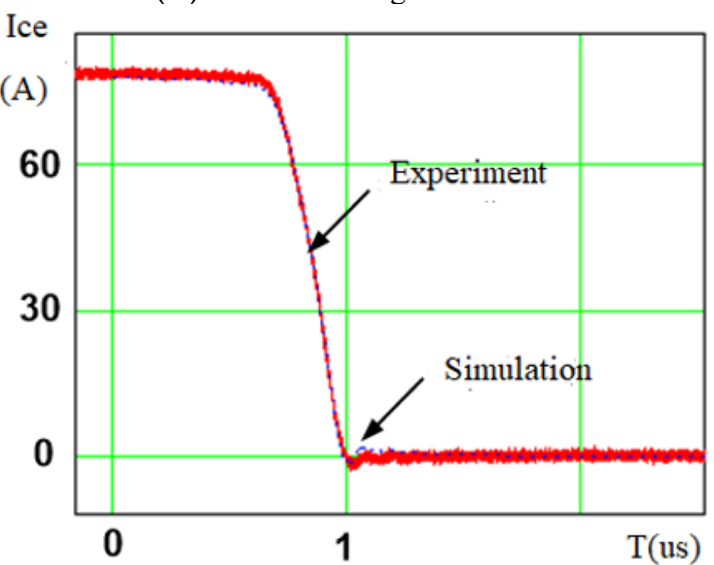

(b) Turn-off current waveform

Fig5. Comparison of shutdown waveforms

It can be seen from Figure 5 that the IGBT model considering trench gate structure is basically consistent with the experimental waveform.

The error causes are analyzed as follows:

(1) The gate drive circuit, load circuit and stray inductance of the device itself are difficult to be accurate;

(2) It is difficult to unify the average effect of carrier lifetime in the model with the dispersion in practical work;

(3) It is difficult to give consideration to the idealization and uniqueness of structural parameters and the dispersion of device technology in model simulation;

(4) Reasonable assumptions and simplification in the modeling process;

(5) Errors introduced in the process of experimental testing.

\section{Summary}

Compared with the traditional planar gate structure, this paper divides the base region into PNP and PIN according to the characteristics of carrier movement in the trench gate structure, and then analyzes the carrier transport and current in the base region according to whether the depletion layer of PNP can cover the trench of PIN, which reflects the characteristics of trench gate structure.

\section{Acknowledgments}

This work was supported by the 2018 year Youth Foundation of Wuhan Donghu University(2018dhzk005), and the Scientific Research Project of Hubei Education Department in 2019(B2019267).

\section{References}

1. Chen Xudong, Cheng Jianbing, Teng Guobing, et al. Novel trench gate field stop IGBT with trench shorted anode[J]. Journal of Semiconductors, 2016, 37(5): 6164.

2. Qin Mengliang, Li Zehong, Zhang Bo, et al. Trench gate IGBT structure with floating P region[J]. Journal of Semiconductors, 2010,31(2):11-13.

3. Fu Qiang, Zhang Bo, Luo Xiaorong, et al. A dual-gate and dielectric-inserted lateral trench insulated gate bipolar transistor on a silicon-on-insulator substrate[J]. Chinese Physics B, 2013,22(7):1-5.

4. Bryant A T, Liu Liqing, Santi E, et al. Modeling of IGBT resistive and inductive turn-on behavior[J]. IEEE Transactions on Industry Application, 2008,44(3):904-914.

5. Iannuzzo F, Busatto G. Physical CAD model for high voltage IGBTs based on lumped-charge approach[J]. IEEE Transactions on Power Electron, 2004,19(4):885-893.

6. Udrea F, Chan S, Thomson J. Development of the next generation of insulated gate bipolar transistors based on trench technology[C]. The $27^{\text {th }}$ European Solid-State Device Research Conference,1997:504507. 